\title{
Insights from the docked DoxDA Model with Thiosulphate
}

\author{
Sujay Ray \& Angshuman Bagchi *
}

Department of Biochemistry and Biophysics, University of Kalyani - 741245, Nadia, West Bengal, India, Angshuman Bagchi E-mail: angshumanb@gmail.com; *Corresponding author

Received February 25, 2016; Accepted March 30, 2016; Published April 10, 2016

\begin{abstract}
:
Redox reaction of inorganic sulphur compound is very essential to maintain a global sulphur cycle. Certain experimental evidences suggest that gamma-proteobacterial Acidothiobacillus thiooxidans; lacking the sulphur-oxidizing (sox) operon, has an alternative thiosulphate oxidation pathway. Dox operon having essentially participating proteins; DoxD and DoxA serves as the central players for this alternative pathway of thiosulphate oxidation. So, to identify their role in thiosulphate oxidation process, functional 3D model of DoxD and DoxA protein's independently functioning conserved domains were built after the contentment of necessary stereochemical features. After formation of the best suited DoxDA protein-complex, DoxDA was MD simulated in several steps and finally through MD simulation run utilizing GROMACS. Even after running beyond 20ns, 18ns simulated protein complex was the most stable and was selected for further study. Residual binding mode conferred mainly two ionic and twelve Hbonded interactions in DoxDA. Astonishingly, Asp167 and Arg18 from DoxA and DoxD, respectively was observed to hold a pivotal role in $6 \mathrm{H}$-bonds accompanied by a separate ionic interaction. Interestingly, four residues from DoxD; Trp32, Met33, Lys36 and Asn140 strengthened the DoxD-thiosulphate interaction. Interaction energy $(\Delta G=(-) 222.016 \mathrm{kcal} / \mathrm{mol})$ and net solvent accessibility calculations depicts spontaneous and fervent residual participation in DoxDA, which is essential for thiosulphate interaction and further sulphur oxidation. Conformational flexibility in DoxD with increased coil percentage benefits DoxD and makes its susceptible for the interaction with thiosulphate even after spontaneous interaction with DoxA. Therefore, this study serves as an insight at computational basis for sulphur oxidation even in organisms lacking sox operon.
\end{abstract}

Keywords: Varied Molecular Modeling, Molecular Dynamics Simulated Protein-Protein Residual Participation, Protein-Ligand Interactions, Energy Estimation, Conformational Fluctuations and Alternative Sulphur Oxidation Pathway.

\section{Background:}

Hydrogen sulfide is typically unfavorable to various plants and creature tissues. At whatever point sulfide is produced, it is a prime need to oxidize the sulfide to essential sulfur [1]. Thiosulfate is the main sulfur substrate that is all around oxidized by dominant part of known chemolithotrophic life forms [1]. In our surroundings, a standout amongst the most imperative arrangements of biogeochemical responses are the redox reactions of environmental sulfur compounds and their steady turnover is highly important to adjust a biosystem with sulfur compounds [1]. With a broad range of oxidation states, sulfur gets oxidized variedly via the utilization of the respiratory electron transport chains [2]. This varied oxidization methods for sulphur is carried out by a wider and sundry faction of micro-organisms. These microorganisms are documented to maintain a specific gene family for the purpose, which is known as the sox operon [2]. This operon includes soxVW succeeded by soxXYZABCDEFGH [2]. Earlier experimentation involving in silico approaches were dealt to uncover the computational biochemistry for the oxidation of sulfur anion in distinct life forms like Paracoccus pantotrophus and others [3].

However, not every organism has the entire sox operon crucial for complete sulfur oxidation. Acidophilic chemoautolithotrophs gamma-proteobacteria such as Acidithiobacillus thiooxidans has incomplete sox operon and therefore, should oxidize thiosulfate with an alternate means [4, 5]. Thus, it utilizes dox operon for the process. Generally, thiosulfate at the periplasm is synthesized impulsively from a sulfur atom and 
sulfite. It further leads to the preparation of tetrathionate via thiosulfate quinone oxidoreductase (TQO). TQO complex is encoded by $\operatorname{doxDA}$ in which the larger subunit (DoxD) forms tetrathionate from thiosulfate and instantaneously liberates two electrons [4, 5]. Simultaneously, the smaller subunit; DoxA transfers these liberated electrons to quinone $[4,5]$. The hydrolysis of tetrathionate is succeeded with the help of TetH; tetrathionate hydrolase (a soluble protein on the periplasm), to generate thiosulfate $[4,5]$.

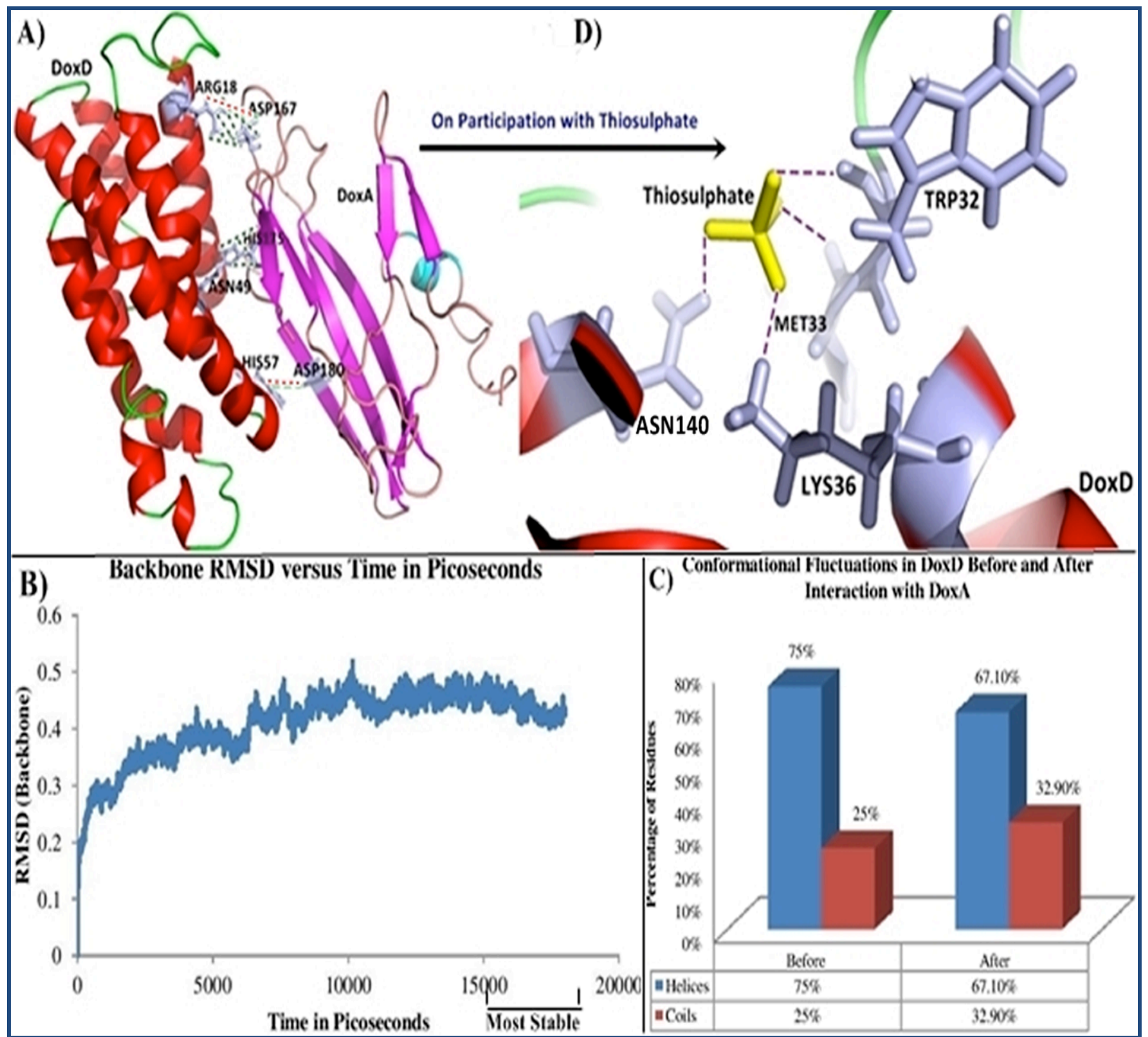

Figure 1: A) 3D structural demonstration of DoxD and DoxA in their interactive state from the most stable simulated complex. Red shaded helices with green interspersing coils represent DoxD protein, whereas; cyan shaded helices, magenta shaded sheets with interspersing pink shaded coils represent the 3D structure of DoxA protein. The interactive residues are shown in blue-white sticks with H-bonded interactions in deep-green dashed lines and ionic interactions in red shaded dashed lines; B) The backbone RMSD fluctuation in DoxDA protein complex showing 18000 picoseconds (18ns) complex structure to be the most stable one; C) The increased percentage of coils and detailed conformation fluctuations in DoxD after interaction with DoxA; D) The residual participation of DoxD to interact with thiosulphate (yellow) with residues represented in blue-white sticks and purple dashes represent the interaction.

So to reveal the detail biochemistry of sulphur oxidation at molecular level DoxDA complex formation, their binding mode and interaction with thiosulphate serve as the essential necessity. Earlier, no investigations dealt with the molecular level documentation of the entire interaction pattern, alterations in conformational stability of DoxDA and also its participation 
with thiosulphate. Thus, the present study concentrated on the 3-dimensional (3D) auxiliary portrayal of the fundamental Dox proteins (DoxD and DoxA) from Acidithiobacillus thiooxidans. Protein-protein and protein-ligand docking studies were performed to acquire the necessary and decisive DoxDA and DoxDA-thiosulphate complex. The protein-protein and proteinligand complex models underwent optimizations and simulations to accomplish a steady interactive conformation. Only after that, the relevant responsible residues for each of the interactive complexes were perceived and reported, specifically. Furthermore, the conformational variations in DoxD protein due to DoxA interaction were analyzed after evaluation. The residues and binding patterns for sulfur oxidation by the interaction of DoxD and thiosulphate was also disclosed. Therefore, this probe aids to discern the detailed alternative pathway with a molecular level approach for sulfur oxidation in microorganisms devoid of possessing the sox operon.

Nevertheless, the process of sulfur oxidation through sox operon in $\alpha$-proteobacterial and gamma-proteobacterial species as well as molecular basis of interaction for removal of other environmental pollutants was illustrated previously [3]. Interestingly, every gamma-proteobacterial species is documented not to possess the sox operon system completely. Therefore, the substituent helper dox operon aids in the entire sulfur oxidation phenomena in such species. This instigates to scrutinize into the details of the residual revelation involving the biochemistry of sulfur oxidation mechanism in Acidithiobacillus thiooxidans. It further enables to examine and expose the residual and molecular level biochemistry for specific reactions for the oxidation of the anionic form of sulfur from the environment. This study therefore, may serve to be beneficial in the accomplishment of the hitherto unexplored molecular phenomena for oxidation reaction of the anionic sulfur, specifically via dox operon system in the gammaproteobacterial species, like Acidithiobacillus thiooxidans.

\section{Methodology:}

Molecular Modeling of DoxD and DoxA Proteins

The amino acid sequences of DoxA and DoxD of Acidithiobacillus thiooxidans were obtained from NCBI (Accession no.: WP_010638552.1). The conserved domain regions in both the proteins were identified using Pfam search and were extracted for building their 3D structures. The template search for either of the proteins against PDB [6] using PSI-BLAST [7], yielded no suitable templates for performing homology modeling.

Fold-recognition approach via I-TASSER was utilized to built the 3D structure of DoxD [8]. No suitable structure of DoxA was determined using fold-recognition. So, ab-initio model building algorithm; QUARK [9] was utilized for generation of de novo 3D model of DoxA.

\section{Energy Minimization and Validation of Modelled Structure}

Distorted loop regions of the modelled proteins were optimized using ModLoop [10]. Thus overall energy of the modelled proteins was minimized by this technique. Energy minimized structures were subjected to validate using Verify3D [11] and
Ramachandran plot [12]. Zero amino acid residues were perceived to be disallowed region in Ramachandran Plot.

\section{Molecular Docking Studies between DoxD and DoxA from Acidithiobacillus thiooxidans}

Residual binding mode between DoxD and DoxA proteins was calculated with the aid of ClusPro2.0 [13]. Model 0 binding pose was taken for our calculation as it was the best cluster size among others.

\section{Molecular Dynamics Simulation of Docked Protein Complex}

For MD simulation of DoxDA, initially native structure was reached using Fragment-Guided MD simulation (FGMD) [14] followed by utilization of Chiron [15]. This native complex structure was further subjected to 20ns MD simulation using GROMACS [16] in a periodic environment. Backbone RMSD of the protein structure was plotted against time to identify stable conformation in the simulation run. The 18 ns structure was taken for further study for being the most stable one

\section{Analysis of Interactive Residues and Binding Patterns in DoxDA}

Protein interaction calculator (P.I.C) helped to calculate residual participation for protein-protein interaction from the stable 18ns complex structure [17]. Ionic bonds and hydrogen bonding interactions were mainly focused for protein structural stability. Binding-site analyse tool of Discovery Studio (DS) was utilized for getting consensus outcomes.

\section{Stability Estimations and Conformational Transition in DoxD due to DoxA Interaction}

Three-state conformational adaptability towards helices, sheets and coils in DoxD, before and after interaction with DoxA was calculated using DSSP method [18]. To evaluate the spontaneous interaction and stability in interaction of DoxD and DoxA, the interaction energy (via DS from Accelyrs) and net area for solvent accessibility (ASA) was estimated. Interaction is spontaneous for high negative $\Delta G$ value. Reduction in net ASA value depicts more surface residues to participate in the interaction pattern [19].

\section{Interaction of Thiosulphate-DoxDA and Analysis of Residual Participation}

To understand thiosulphate oxidation, protein-ligand docking via utilization of AutoDock Vina software [20] was performed. The PDBQT files of DoxDA (receptor) and thiosulphate (ligand) were utilized to obtain the best binding complex (thiosulphateDoxDA) based on the highest interaction energy (lowest $\Delta G$ value). Residual contribution for $\mathrm{H}$-bonds in thiosulphateDoxDA was calculated using PyMOL.

\section{Results \& Discussion:}

This study explores the structural features of two competent proteins for sulphur oxidation; DoxD and DoxA. Further their binding patterns and their involvement in thiosulphate oxidation through molecular modelling and docking based approach from a gamma proteobacterial Acidithiobacillus thiooxidans was explored into. Domains being the most conserved regions and the regions that are capable of their efficient function independently were identified for DoxD and DoxA. As no suitable templates for performing homology 
modelling was obtained, approach for two advanced protein modelling techniques such as fold recognition and ab-initio modelling were used to model DoxD and DoxA proteins. For the precision analysis of the de novo modelled protein; DoxA, TM-score was calculated which yielded to $0.3124 \pm 0.0833$. Their stereo-chemical properties were observed to be satisfactorily satisfied with $90.7 \%$ and $90.4 \%$ residues in core region of the Ramachandran plot for DoxD and DoxA, respectively with zilch residues in disallowed regions. The satisfactorily modelled model DoxD and DoxA proteins were observed to be 164 and 138 amino acid residues long.

\begin{tabular}{|c|c|c|c|c|c|c|}
\hline \multirow{4}{*}{ Main Chain-Side Chain Interactions } & Residue Number & Protein & Residue & Residue Number & Protein & Residue \\
\hline & 175 & A & HIS & 7 & $\mathrm{D}$ & GLN \\
\hline & 289 & A & GLN & 42 & $\mathrm{D}$ & PRO \\
\hline & 289 & A & GLN & 42 & $\mathrm{D}$ & $\mathrm{PRO}$ \\
\hline \multirow{10}{*}{ Side Chain-Side-Chain Interactions } & Residue Number & Protein & Residue & Residue Number & Protein & Residue \\
\hline & 175 & A & HIS & 49 & $\mathrm{D}$ & ASN \\
\hline & 18 & $\mathrm{D}$ & ARG & 167 & A & ASP \\
\hline & 18 & $\mathrm{D}$ & ARG & 167 & A & ASP \\
\hline & 18 & $\mathrm{D}$ & ARG & 167 & A & ASP \\
\hline & 18 & $\mathrm{D}$ & ARG & 167 & $\mathrm{~A}$ & ASP \\
\hline & 18 & $\mathrm{D}$ & ARG & 167 & A & ASP \\
\hline & 18 & $\mathrm{D}$ & ARG & 167 & A & ASP \\
\hline & 49 & $\mathrm{D}$ & $\mathrm{ASN}$ & 175 & $\mathrm{~A}$ & HIS \\
\hline & 49 & $\mathrm{D}$ & ASN & 175 & A & HIS \\
\hline \multirow{3}{*}{ Ionic-Ionic Interactions } & Residue Number & Protein & Residue & Residue Number & Protein & Residue \\
\hline & 167 & A & ASP & 18 & $\mathrm{D}$ & ARG \\
\hline & 180 & A & ASP & 57 & $\mathrm{D}$ & HIS \\
\hline
\end{tabular}

Table 1 H-Bonded Interactions and Ionic Interactions in Stable Simulated DoxDA protein complex

The structure of DoxD mostly comprises 7 helices interconnected with coils (Figure 1A). Furthermore, the conformational adaptability of DoxA towards helix, sheet and coil is $5.8 \%, 47.1 \%$ and $47.1 \%$, respectively (Figure 1A). The overall protein structure was energy minimized to overcome irregularities in backbone phi and psi angles and thereby attain a stable 3D structure. These stable 3D structures of the two proteins were docked to form the DoxDA complex and were then subjected to MD simulation to get the best stable interactive complex structure. Figure 1B, it was observed that within 15ns - 18ns time-step there was no major fluctuation in backbone RMSD, so 18ns simulated structure was selected for further study. To analyze the residual contribution from the respective positions in either of the two proteins, the chiefly contributing ionic interactions yielded that negatively charged; Asp167 and Asp180 from DoxA protein formed two firm ionic interactions with positively charged; Arg18 and His57 of DoxD protein (Table 1). Figure 1A shows the ionic interactions in red shaded dashed lines. Additionally, 3 main-chain_side-chain interactions and 9 side-chain_side-chain H-bonds aided the interaction of DoxD and DoxA, more potently (Table 1). Arg18 from DoxD and Asp167 from DoxA formed solely 6 sidechain_side-chain H-bonds, accompanied by an ionic interaction (Figure 1A). The other three side-chain_side-chain interactions were accomplished by solely Asn 49 from DoxD and His175 from DoxA proteins. Figure 1A shows the H-bonds in deepgreen dashed lines. Altogether, a cavity-like structure is prepared for DoxA to fit efficiently into the partner DoxD protein. Therefore, these two residues from the respective proteins hold a pivotal role to strengthen the interaction among DoxD and DoxA.

Further, interaction energy in DoxDA protein complex calculated using CHARMM force-field via Discovery Studio packages yielded $\Delta \mathrm{G}$ value to $-222.016 \mathrm{kcal} / \mathrm{mol}$. Net accessible surface area (ASA) upon DoxD decreased from $9269.39 \AA^{2}$ (before interaction) to $9198.07 \AA^{2}$ after interaction with DoxA. Overall, these outcomes conferred stronger and spontaneous interaction of DoxD with DoxA with sufficient participation of residues.

The structural transitions in DoxD after interaction with DoxA depicted that there was an abrupt ascent in the percentage of residues forming flexible coil-like conformation (from $25 \%$ to $32.9 \%$ ) to eagerly interact with DoxA (Figure 1C). This therefore shows that DoxD protein was susceptible to interact with thiosulpahte even after instantaneous and spontaneous interaction with DoxA. Thereafter, thiosulphate and DoxDA interaction was focussed upon to observe the proficient binding pattern in the protein and the ligand for effective thiosulphate oxidation in sox operon lacking; Acidithiobacillus thiooxidans. From DoxD, mainly four amino acid residues; Asn140 and three adjacent residues; Met33, Trp32 and Lys36 formed stronger H-bonds with thiosulphate (Figure 1D). The H-bonds in DoxD-thiosulphate were shown with purple dashes in Figure 1D.

Therefore, to summarize, this present study analyzed vividly the detailed residual interaction and binding patterns for stronger and random interaction between DoxD, DoxA and thiosulphate for the oxidation of thiosulphate in sox operon lacking microorganisms like from Acidithiobacillus thiooxidans. The detailed structural biochemistry and molecular-level study with evaluation of interaction energy and net solvent accessible area also aided to examine the spontaneous and active participation of residues from the two proteins for oxidising sulphur and rendering a sulphur-free environment, even by the participation of sox operon lacking microorganisms. Thus, it 
creates a rationale for sulphur oxidation and removal of harmful pollutants like sulphides with the involvement of sox operon lacking microorganisms. It therefore opens-up a future scope to study the mutation in either of the Dox proteins and further their impact upon sulphur oxidation phenomena.

\section{Conclusion and Future Scope}

Concisely, this complete study describes mainly the interplay between two important essential proteins; DoxD and DoxA from gamma-proteobacterial Acidothiobacillus thiooxidans and thiosulphate for maintenance of the element sulphur, globally. 3D functional model of conserved domains of DoxD and DoxA were analyzed and demonstrated, followed by energy minimization. Complex formation of DoxDA was followed by MD simulation run. During simulation run, the stable complex structure was obtained at $18 \mathrm{~ns}$ time-step, though simulation was performed beyond 18ns time-step too. Residual revelations disclosed essentially 2 sets and 12 sets of ionic and H-bonded interactions. Asp167 from DoxA and Arg18 from DoxD played pivotal role for DoxDA interaction with a joint participation into 6 side-chain_side-chain interactions accompanied by ionic interactions. Conformational fluctuation in DoxD due to DoxA interaction affirmed the increased flexibility of DoxD to interact with DoxA. Interaction energy and net ASA calculations showed spontaneous and active participation of residues for firmer DoxDA interaction. Four residues from DoxD were observed to participate essentially for thiosulphate interaction. Though there exists former investigation for sox operon and participation of Sox proteins for thiosulphate oxidation, but hitherto interactive details of DoxDA complex for thiosulphate oxidation were not explored. Therefore, this study illustrates the detailed molecular basis for the interaction pathway in organisms lacking sox operon but essentially participating in sulphur oxidation phenomena. Therefore, it instigates a novel outlook for sulphide-free atmosphere in absence of sox operon. Future research arena lies in examination of the mutational impacts in the oxidation phenomena upon mutations in Dox proteins.

\section{Acknowledgement:}

Deep gratitude is extended to Bioinformatics Resources and Applications Facility (BRAF), C-DAC, Pune for the necessary support. Authors are indebted to Department of Biochemistry and Biophysics, University of Kalyani for providing essential infrastructural support.

\section{References:}

[1] Ogawa T et al. Biosci Biotechnol Biochem. 2010 74: 771 [PMID: 20378984]

[2] Appia-Ayme C et al. Journal of Bacteriology 2001 183: 6107 [PMID: 11567011]

[3] Bagchi A \& Roy P, Biochem Biophys Res Commun. 2005 331: 1107 [PMID: 15882991]

[4] Fazzini R et al. Biotechnology and Bioengineering 2013 110: 2242 [PMID: 23436458]

[5] Mangold S et al. Microbiol. 2011 2: 17 [PMID: 25511286]

[6] Berman HM et al. Nucleic Acids Research 2000 28: 235 [PMID: 1059223]

[7] Altschul SF et al. Journal of Molecular Biology 1990 215: 403 [PMID: 2231712]

[8] Roy A et al. Nature. Protocol 2010 5: 725 [PMID: 20360767]

[9] Xu D \& Zhang Y, Proteins 2012 80: 1715 [PMID: 22411565]

[10] Fiser A \& Sali A, Bioinformatics 2003 19: 2500 [PMID: 14668246]

[11] Eisenberg D et al. Methods Enzymol. 1997 277: 396 [PMID: 9379925]

[12] Ramachandran GN \& Sashisekharan V, Adv. Protein Chem. 1968 23: 283 [PMID: 4882249]

[13] Comeau SR et al. Bioinformatics 2004 20: 45 [PMID: 14693807]

[14] Zhang J et al. Structure 2001 19: 1784 [PMID: 22153501]

[15] Ramachandran S et al. Bioinformatics 2011 79: 261 [PMID: 21058396]

[16] Hess B et al. J Chem Theory Comput. 2008 4: 435 [PMID: 26620784]

[17] Tina KG, Nucleic Acids Research. 2007 35: W473 [PMID: 17584791]

[18] Kabsch W \& Sander C, Biopolymers. 1983 22: 2577 [PMID: 6667333]

[19] Gerstein M, Acta Cryst. 1992 48: 271 DOI: 10.1107/ S0108767391012680

[20] Trott O \& Olson AJ, J Comput Chem. 2010 31: 4 [PMID: 19499576]

Edited by $P$ Kangueane Citation: Ray \& Bagchi, Bioinformation 12(2): 69-73 (2016)

License statement: This is an Open Access article which permits unrestricted use, distribution, and reproduction in any medium, provided the original work is properly credited. This is distributed under the terms of the Creative Commons Attribution License

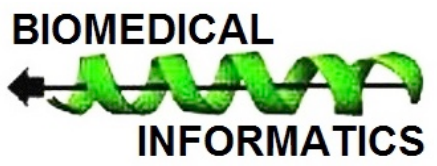

